\title{
Neoadjuvant versus adjuvant management of resectable pancreas cancer: A review of the literature
}

\author{
Konstantin Schlick
}

Received: 16 June 2021 / Accepted: 4 August 2021 / Published online: 8 September 2021

(C) The Author(s) 2021

\begin{abstract}
Summary Neoadjuvant therapy is well-accepted in the treatment of borderline resectable and locally advanced PC, the benefit of neoadjuvant chemotherapy in patients with resectable disease, however, is currently not so clear. Here we provide an up date on the literature.
\end{abstract}

Keywords Pancreatic cancer · Surgery · Outcome · Neo-adjuvant chemotheray

Pancreatic cancer (PC) is currently associated with one of the lowest survival rates among malignancies with a 5-year overall mortality rate of $95 \%$ [1]. The incidence rate almost mirrors the mortality rate, since prognosis is dismal and has not changed over the last few decades although novel therapy strategies have been implemented in clinical routine [2]. The only potential chance for cure remains radical tumor resection [3]. However, only 15-20\% of all newly diagnosed patients are potential candidates for surgical resection, resulting in a median survival of up to 24 months [4]. Inoperable, locally advanced and metastatic disease are associated with significantly shorter survival [5]. The 5-year overall survival (OS) rate remains poor for nonsurgery candidates at $12 \%$ and $3 \%$ for locally advanced and metastatic disease, respectively [6].

While the use of neoadjuvant therapy is wellaccepted in the treatment of borderline resectable and locally advanced PC, the benefit of neoadjuvant chemotherapy in patients with resectable disease has been a topic of debate.

PD OA Dr.med.univ. K. Schlick (凶)

IIIrd Medical Department with Hematology \& Medical Oncology, Hemostaseology, Rheumatology \& Infectious Diseases, Oncologic Center, Paracelsus Medical University Salzburg, Müllner Hauptstrasse 48, 5020 Salzburg, Austria k.schlick@salk.at
Neoadjuvant therapy in the resectable disease setting has known benefits. The prioritization of systemic therapy in patients who suffer from micrometastatic disease may have the potential to expand the population of patients undergoing surgical resection to those with favorable tumor biology. Patients may also derive benefit from a seemingly increased tolerability of multidrug regimens like fluorouracil, leucovorin, irinotecan, and oxaliplatin (FOLFIRINOX) and gemcitabine with nab-paclitaxel administered in the neoadjuvant setting, compared to the adjuvant setting [7]. Finally, sequencing chemotherapy before surgery delivers invaluable individualized prognostic information on the effectiveness of chemotherapy with key histologic markers available from the surgical specimen including microscopic margin status, nodal metastases, and tumor regression grade; however, prospective trials, institutional series, and large cancer registries consistently report that up to $50 \%$ of patients are unable to receive adjuvant therapy-generally due to early cancer recurrence or poor performance status after surgery. A greater number of individuals are unable to complete all intended cycles of adjuvant therapy [8]. For example, a recent study of Medicare patients found that only $7 \%$ were able to receive all intended adjuvant chemotherapy [9].

Furthermore, the delivery of neoadjuvant therapy affords an important test of tumor biology to rule out rapid disease progression, especially in light of an indeterminate radiographic finding (e.g., liver or lung lesion) or elevated CA 19-9, which can potentially help avoid the morbidity of a nontherapeutic pancreatectomy.

The randomized phase III PREOPANC-1 trial compared preoperative chemoradiotherapy and upfront surgery for patients with borderline resectable and resectable PC [10]. Notably, this was the first large- 
scale trial with accrual completion that reported results evaluating patients with resectable PC.

Patients enrolled in the study were randomized to either preoperative gemcitabine-based chemoradiotherapy followed by surgery and adjuvant gemcitabine chemotherapy or upfront surgery followed by adjuvant gemcitabine chemotherapy. In total, 248 patients were randomized to treatment. After a median follow-up period of 27 months, there was no significant difference in OS in patients who were randomized to undergo preoperative therapy (16.0 months; 95\% confidence interval [CI] 13.0-20.9) compared to patients who were randomized to immediate surgery (14.3 months; 95\% CI 12.7-17.9), which was the primary endpoint for the study. However, there were clear benefits demonstrated in terms of OS, diseasefree survival (DFS), and rate of margin negative resection in the predefined analysis of patients with borderline resectable disease. These outcomes support the premise that preoperative chemoradiation enhances locoregional disease control and is preferentially advantageous in patients with increasing vascular involvement that pose a greater risk for margin-positive resection. However, the applicability of results highlighting the use of single-agent gemcitabine adjuvant therapy is limited in the current era.

In order to address the issues of novel chemotherapeutic regiments in peri-operative setting in more detail, two trials are currently investigating FOLFOX/ FOLFIRINOX in the Panache Trial (upfront surgery and adjuvant chemotherapy vs perioperative FOLFOX/ FOLFIRINOX) and neoadjuvant Folfirinox vs perioperative gemcitabine + RTX in resectable and borderline resectable PDAC in the Preopanc-2 trial.

The Japanese randomized PREP-02/JSAP05 trial compares neoadjuvant chemotherapy with gemcitabine and S-1, an oral 5-fluorouracil derivative with immediate surgery in patients with potentially resectable PC [11]. The median OS in neoadjuvant treated patients was 36.7 months, compared to 26.6 months in patients who underwent upfront surgery ( $p=0.015$; hazard ratio $[\mathrm{HR}] 0.72 ; 95 \% \mathrm{CI}$ $0.55-0.94)$. However, there was a significant rate of grade 3 and grade 4 adverse events (48.8 and 23.8\%, respectively) in patients treated with neoadjuvant therapy. Despite this, neoadjuvant chemotherapy led to a decreased rate of lymph node metastases compared to patients who underwent upfront surgery (59.6 vs. $81.5 \% ; p<0.01$ ) and did not lead to worse postoperative outcome. Taken together, the JSAP-05 trial is the first multi-institutional phase III trial that demonstrates that the use of neoadjuvant chemotherapy leads to an improvement in OS in patients with primary resectable PC.

These results have a somewhat restricted application in Europe and North America, where the oral fluoropyrimidine $S-1$ is not available.

SWOG S1505, a randomized phase II trial, evaluates patients, with resectable PC, randomized to either perioperative mFOLFIRINOX or perioperative gemcitabine/nab-paclitaxel with a planned 12 weeks of neoadjuvant chemotherapy in each arm, followed by surgical resection, and then by 12 weeks of adjuvant chemotherapy of the same type [12].

Patients treated in the mFOLFIRINOX arm had a median OS of 22.4 months, while patients in the gemcitabine/nab-paclitaxel arm had a median OS of 23.6 months. The primary endpoint did not meet the prespecified statistical threshold in either arm of the trial. While preoperative therapy in this trial led to high rates of surgical resection, with margin-negative resection performed in $85 \%$ of patients in each arm, the modest survival outcomes reported in this trial are likely contributed to the low percentage of patients completing the whole protocol-defined therapy.

The ALLIANCE A021806 trial seeks to definitively clarify the role of neoadjuvant chemotherapy in patients with resectable PC. This trial has recently opened for accrual in July of 2020 and aims to randomize patients with resectable PC to perioperative chemotherapy or up-front surgery and adjuvant chemotherapy with mFOLFIRINOX.

\section{Conclusion}

- Currently neo-adjuvant chemotherapy in resectable pancreatic cancer is not endorsed in clinical guidelines, due to the lack of randomized phase III trials.

- Ongoing and future trials aim to further clarify the role of neoadjuvant treatment in patients who present with primary resectable disease.

- Future research is needed to establish predictive biomarkers, measures of therapeutic response, and multidisciplinary strategies to improve patient-centered outcomes.

Funding Open access funding provided by Paracelsus Medical University.

Conflict of interest K. Schlick declares that he has no competing interests.

Open Access This article is licensed under a Creative Commons Attribution 4.0 International License, which permits use, sharing, adaptation, distribution and reproduction in any medium or format, as long as you give appropriate credit to the original author(s) and the source, provide a link to the Creative Commons licence, and indicate if changes were made. The images or other third party material in this article are included in the article's Creative Commons licence, unless indicated otherwise in a credit line to the material. If material is not included in the article's Creative Commons licence and your intended use is not permitted by statutory regulation or exceeds the permitted use, you will need to obtain permission directly from the copyright holder. To view a copy of this licence, visit http://creativecommons.org/licenses/by/4.0/. 


\section{References}

1. Miller KD, Nogueira L, Mariotto AB, et al. Cancer treatment and survivorship statistics. CA Cancer J Clin. 2019;69(2019):363-85.

2. Tesfaye AA, Kamgar M, Azmi A, et al. The evolution into personalized therapies in pancreatic ductal adenocarcinoma: challenges and opportunities. Expert Rev Anticancer Ther. 2018;18:131-48.

3. Siegel RL, Miller KD, Jemal A. Cancer statistics. CA Cancer J Clin. 2019;69(2019):7-34.

4. Bilimoria KY, Bentrem DJ, Ko CY, et al. National failure to operate on early stage pancreatic cancer. Ann Surg. 2007;246:173-80.

5. He J, Edil BH, Cameron JL, et al. Young patients undergoing resection of pancreatic cancer fare better than their older counterparts. J Gastrointest Surg. 2013;17:339-44.

6. Jin J, Teng C, Li T. Combination therapy versus gemcitabine monotherapy in the treatment of elderly pancreatic cancer: a meta-analysis of randomized controlled trials. Drug Des Devel Ther. 2018;12:475-80.

7. Neoptolemos JP, Palmer DH, Ghaneh P, et al. Comparison of adjuvant gemcitabine and capecitabine with gemcitabine monotherapy in patients with resected pancreatic cancer (ESPAC-4): a multicentre, open-label, randomised, phase 3 trial. Lancet. 2017;389:1011-24.

8. Valle JW, Palmer D, Jackson R, et al. Optimal duration and timingofadjuvantchemotherapyafterdefinitivesurgeryfor ductal adenocarcinoma of the pancreas: ongoing lessons from theESPAC-3 study. JClin Oncol. 2014;32:504-12.

9. Altman AM, Wirth K, Marmor S, et al. Completion of adjuvant chemotherapy after upfront surgical resection for pancreatic cancer is uncommon yet associated with improved survival. Ann Surg Oncol. 2019;26:4108-16.

10. Versteijne E, Suker M, Groothuis K, et al. Preoperative chemoradiotherapy versus immediate surgery for resectable and borderline resectable pancreatic cancer: results of the dutch randomized Phase III PREOPANC Trial. JClin Oncol. 2020;38:1763-73.

11. Motoi F, Kosuge T, Ueno H, et al. Randomized phase II/III trial of neoadjuvant chemotherapy with gemcitabine and S-1 versus upfront surgery for resectable pancreatic cancer (Prep-02/JSAP05). Jpn J Clin Oncol. 2019;49:190-4.

12. Ahmad SA, Duong M, Sohal DPS, et al. Surgical Outcome Results From SWOG S1505: A Randomized Clinical Trial of mFOLFIRINOX Versus Gemcitabine/Nab-paclitaxel for Perioperative Treatment of Resectable Pancreatic Ductal Adenocarcinoma. Ann Surg. 2020;272:481-6.

Publisher's Note Springer Nature remains neutral with regard to jurisdictional claims in published maps and institutional affiliations.

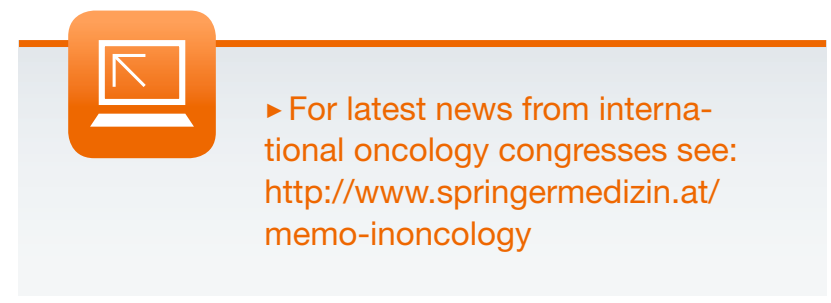

